\title{
Poster Abstract: A Data-Driven System for City-Scale Personal Energy Footprint Estimations
}

\author{
Peter Wei \\ Columbia University \\ pw2428@columbia.edu
}

\begin{abstract}
Energy footprinting has the potential to raise awareness of energy consumption and lead to energy saving behavior. However, current methods for estimating energy consumption cannot provide fine enough temporal or spatial granularity for a reasonable personal energy footprint estimate. In this work, we present a data-driven system design for estimating personal energy footprint in the city in real-time, even in built environments that do not have existing or accessible energy data or population data.
\end{abstract}

\section{CCS CONCEPTS}

- Information systems $\rightarrow$ Geographic information systems; Data streams; Data mining;

\section{KEYWORDS}

Energy Footprint, Data Mining, Geographic Information Systems

\section{ACM Reference Format:}

Peter Wei and Xiaofan Jiang. 2018. Poster Abstract: A Data-Driven System for City-Scale Personal Energy Footprint Estimations. In The 5th ACM International Conference on Systems for Built Environments (BuildSys '18), November 7-8, 2018, Shenzen, China. ACM, New York, NY, USA, 2 pages. https://doi.org/10.1145/3276774.3281018

\section{INTRODUCTION}

Recently, energy consumption awareness has become an important area of research. In [1], the authors show that certain feedback mechanisms given to occupants raises awareness and can lead to energy saving behavior. One such mechanism is by notifying the occupant in real-time of their numerical energy consumption.

Working systems for direct real-time energy feedback, also called energy footprinting, have been demonstrated in [6] in commercial buildings and recently in [5] for the home environment. However, these systems require infrastructure such as energy monitoring devices for HVAC, lighting, and electric appliances, or localization beacons. For a significant percentage of modern buildings, this infrastructure either does not exist or is inaccessible to the public.

Ideally, an energy footprint measures an occupant's energy consumption throughout the day in all parts of the built environment, including transportation, home and commercial buildings, retail and other communal spaces. In this poster abstract, we present a

Permission to make digital or hard copies of part or all of this work for personal or classroom use is granted without fee provided that copies are not made or distributed for profit or commercial advantage and that copies bear this notice and the full citation on the first page. Copyrights for third-party components of this work must be honored.

For all other uses, contact the owner/author(s).

BuildSys '18, November 7-8, 2018, Shenzen, China

(C) 2018 Copyright held by the owner/author(s)

ACM ISBN 978-1-4503-5951-1/18/11.

https://doi.org/10.1145/3276774.3281018

\author{
Xiaofan Jiang \\ Columbia University \\ jiang@ee.columbia.edu
}

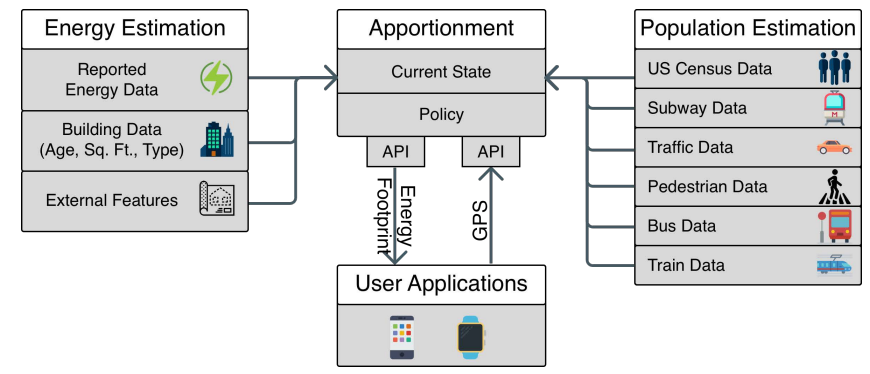

Figure 1: System architecture block diagram.

preliminary system design for energy footprint estimation at the city-scale. Due to the fact that real-time energy consumption and population data may not be available at a granular scale, we propose to utilize a top-down approach starting from city-wide public datasets to generate a personal energy footprint estimate.

\section{SYSTEM DESIGN}

Our system is composed of three connected subsystems: energy estimation, population estimation, and apportionment. The energy and population estimation subsystems provide corresponding estimates for each building, via baseline models constructed from public datasets. The apportionment subsystem uses the energy consumption and population estimates to compute an energy footprint estimate for a user in the building, which is displayed via a mobile application. The system architecture is shown in Figure 1.

New York City is an ideal testbed for this system for two reasons. Firstly, there exist many datasets containing information about various attributes of the city. Energy data collected by energy providers, as well as people counts in different modes of transportation are made publicly available. The abundance of datasets makes possible the study of energy footprinting at a city scale, while presenting new challenges in data representation, cleaning, and modeling.

Secondly, individual energy usage patterns are highly variable in New York City. The age of buildings in New York City varies tremendously, which leads to differences in the efficiency of energy usage. Further, the number of people varies greatly throughout the workday and weekends as people living in areas surrounding Manhattan commute into the city, according to a study done by NYU Wagner [4]. The energy use and population variations makes New York City an ideal testbed for studying different energy footprints.

\subsection{Energy Estimation}

Often, energy consumption data is unavailable at a sufficiently granular level to enable personal energy footprinting. In [3], the authors demonstrate a method for estimating the energy use intensity at the building level. Our system currently utilizes an energy 


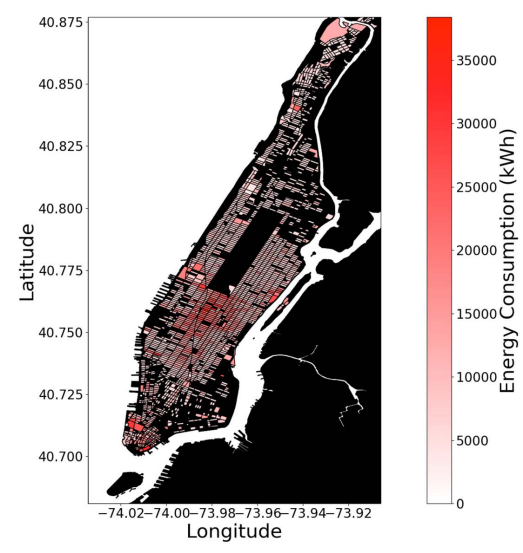

(a) Heatmap of energy consumption in Manhattan by block.

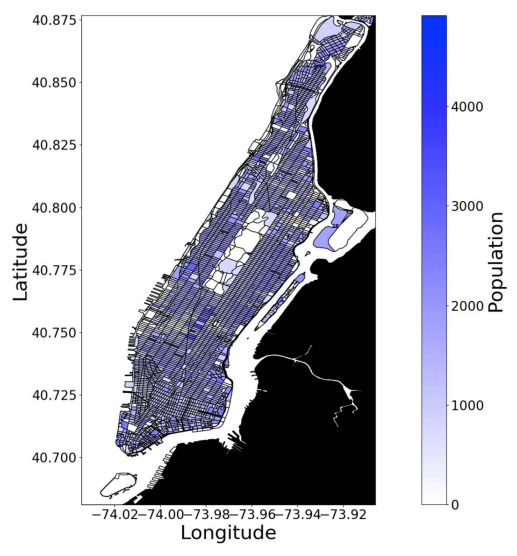

(b) Heatmap of population in Manhattan by block.

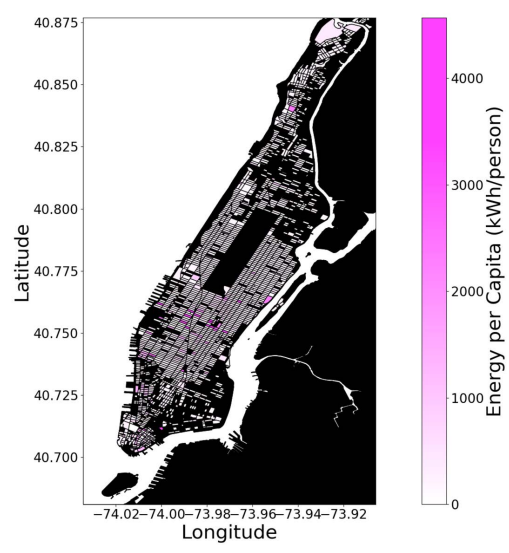

(c) Energy consumption per capita, with a uniform apportionment policy.

Figure 2: Energy Footprinting of Manhattan on a typical weekday at 8 AM.

benchmarking dataset from New York City (Local Law 84) which provides self-reported energy usage intensity (EUI) for buildings over 50,000 square feet. We are also planning to utilize other data sources, such as building characteristics (age, square feet, space usage) to model energy consumption for smaller buildings.

This energy use intensity serves as a baseline energy consumption for a building. An illustration of the average energy consumption by block is shown in Figure 2a. Additional work will focus on modeling changes in energy consumption throughout the day, as well as changes due to weather and other external conditions.

\subsection{Population Estimation}

Currently, there are no public datasets describing population counts at the building level. Further, the population varies throughout the day and week due to the large number of commuters, complicating the task of estimating real-time building population.

However, multiple data sources exist for building a baseline model and real-time estimates. For instance, the Metropolitan Transportation Authority (MTA), which runs the subway system, buses, and train lines in New York City, publishes weekly turnstile data for all subway stations and ridership statistics for stops on the Long Island Railroad. Additionally, the US Census Bureau publishes a baseline population dataset at the block level.

A preliminary baseline model was constructed using the US Census dataset as initial populations for each block. To estimate realtime population dynamics, a population mobility model is utilized for each mode of transportation. As an example, turnstile data from subway stations provide a measure of population inflow and outflow to and from the station's surrounding area. An illustration of the dynamic population is shown in Figure $2 \mathrm{~b}$.

\subsection{Apportionment}

Once an estimate for a building's energy consumption and population are calculated, a user's personal energy footprint can be estimated by applying an apportionment policy. From [2], different apportionment policies can be applied depending on the situation. The simplest apportionment policy is uniform apportionment, such that the total energy consumption is distributed uniformly over the number of people. The energy consumption per capita for a typical morning in Manhattan is shown in Figure 2c.

\subsection{User Applications}

We are currently developing a mobile application for iOS and Android. The application sends GPS data to the server to localize the user to a building; the estimated energy footprint is returned and displayed to the user in real-time. A wearable application is also under development.

\section{CONCLUSIONS AND FUTURE WORK}

In this work, we describe a system design and data-driven approach for personal energy footprinting at the city-scale. Energy consumption data and population data are utilized to form baseline models. In future work, we plan to refine the energy and population models, as well as integrate real-time transit data and external conditions such as weather, to improve energy footprint estimates.

\section{ACKNOWLEDGMENTS}

This research was partially supported by the National Science Foundation under Grant Numbers CNS-1704899 and CNS-1815274.

\section{REFERENCES}

[1] Sarah Darby et al. 2006. The effectiveness of feedback on energy consumption. A Review for DEFRA of the Literature on Metering, Billing and direct Displays 486, 2006 (2006), 26

[2] Simon Hay and Andrew Rice. 2009. The case for apportionment. In Proceedings of the First ACM Workshop on Embedded Sensing Systems for Energy-Efficiency in Buildings. ACM, 13-18.

[3] Constantine E Kontokosta and Christopher Tull. 2017. A data-driven predictive model of city-scale energy use in buildings. Applied energy 197 (2017), 303-317.

[4] Mitchell Moss and Carson Qing. 2012. The Dynamic Population of Manhattan. Rudin Center for Transportation, NYU Wagner School.

[5] Akshay Uttama Nambi SN, Antonio Reyes Lua, Luis Gonzalez, et al. 2018. PEAT, how much am i burning?. In Proceedings of the 9th ACM Multimedia Systems Conference. ACM, 316-327.

[6] Peter Wei, Xiaoqi Chen, Jordan Vega, Stephen Xia, Rishikanth Chandrasekaran, and Xiaofan Jiang. 2017. ePrints: a real-time and scalable system for fair apportionment and tracking of personal energy footprints in commercial buildings. In Proceedings of the 4th ACM International Conference on Systems for Energy-Efficient Built Environments. ACM, 6. 